\title{
Recollection: Élisabeth Allès (1952-2012)
}

\section{Sebastian Veg}

\section{OpenEdition}

\section{Journals}

\section{Electronic version}

URL: http://journals.openedition.org/chinaperspectives/5772

DOI: 10.4000/chinaperspectives.5772

ISSN: 1996-4617

\section{Publisher}

Centre d'étude français sur la Chine contemporaine

\section{Printed version}

Date of publication: 30 March 2012

Number of pages: 2

ISSN: 2070-3449

\section{Electronic reference}

Sebastian Veg, «Recollection: Élisabeth Allès (1952-2012) », China Perspectives [Online], 2012/1 | 2012,

Online since 30 March 2012, connection on 24 September 2020. URL : http://journals.openedition.org/ chinaperspectives/5772 ; DOI : https://doi.org/10.4000/chinaperspectives.5772 
$\mathrm{O}$ ur dear colleague Élisabeth Allès passed away in the early hours of 1 January. Élisabeth was a long-time friend of the CEFC, where she spent two years as a CNRS researcher from 2002 to 2004, and of China Perspectives, as a regular contributor and member of its editorial board. Most recently, she co-edited the special issue entitled "The Deadlock in Tibet" in 2009.

She was an anthropologist and a specialist of Chinese Islam and of Central Asia, in particular of the Dungan culture, the name by which the Chinese communities in Central Asia, mostly made up of Hui Muslims speaking a variant of Gansu dialect, are known. I remember first learning about these communities through a paper she gave at a CEFC conference in Almaty in June 2008, and also the expertise and generosity with which she shared contacts of Muslim Chinese writers and

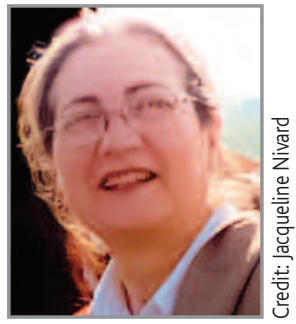
intellectuals with me on this occasion. She published two important articles in China Perspectives on her research, one on the Dungans: "Les Dounganes et la cérémonie du mariage" (1999), and one on education: "Muslim Religious Education in China" (2003). Several articles published in other academic journals are available via her author page on the French Website Persée (link below). She was most recently the director of the EHESS-CNRS China Centre in Paris.

She was also a committed defender of human rights, in particular of Wenzhou immigrants in France, as reflected in our colleague Jean-Philippe Béja's recollection of her, and in the announcement published by the Ligue des droits de l'homme, both linked below.

Sebastian Veg

- Élisabeth Allès' articles on Persée: www.persee.fr/web/revues/home/prescript/author/auteur_oroc_137

- Jean-Philippe Béja's recollection: http://blogs.mediapart.fr/blog/jean-philippe-beja/030112/disparition-d-elisabeth-alles-sinologue-engagee

- Ligue des droits de l'homme's recollection: http://www.ldh-france.org/Hommage-a-Elisabeth-Alles,4016

\section{Book}

Musulmans de Chine. Une anthropologie des Hui du Henan, Paris, EHESS, coll. "Recherches d'histoire et de sciences sociales," 2000,334 pp.

\section{Articles*}

D "Les Dounganes et la cérémonie du mariage: un exemple d'identités multiples," Perspectives chinoises, no. 56, 1999, pp. 62-69.

D "Une autre façon d'être chinois ou le cas des Hui," Hérodote, no. 96, 2000, pp. 117-129.

D "L'islam chinois, unité et fragmentation," together with L. Cherif, C. H. Halfon, Archives en sciences sociales des religions, no. 115, 2001, pp. 15-47 (English translation in Religion, State and Society, vol. 31, no. 1, 2003, pp. 7-35).

D "Quelles normes pour les Chinois musulmans (hui)?," in "La coutume et la norme en Chine et au Japon," ExtrêmeOrient, Extrême-Occident, no. 23, 2001, pp. 53-61.

D "Confucius, Allah et Mao : l'islam en Chine," in A. Feillard (dir.), L'islam en Asie, du Caucase à la Chine, La Documentation française, 2001, pp. 207-239.

D "À propos de l'islam en Chine : provocations antireligieuses et attitudes anticléricales du XIxe siècle à nos jours," in "L'Anticléricalisme en Chine," Extrême-Orient, ExtrêmeOccident, no. 24, 2002, pp. 65-75.

D "L'enseignement confessionnel musulman en Chine," Perspectives chinoises, no. 74, 2002, pp. 21-30.

D "Notes sur quelques relations à plaisanteries entre villages hui et han du Henan," Perspectives chinoises, no. 78, 2003, pp. 49-60.

D "History of Islam in China: Mid-18 $8^{\text {th }}$ to Early $20^{\text {th }}$ Century," in Encyclopedia of Women and Islamic Cultures. Methodologies, Paradigms and Sources, vol. 1, Leiden, Brill, 2003, pp. 120-122.

D "Solidarité religieuse ou culturelle ? Une situation délicate pour les Hui du Xinjiang," in V. Bouillier and C. ServanSchreiber (eds.), De l'Arabie à l'Himalaya. Chemins croisés en hommage à Marc Gaborieau, Maisonneuve et Larose, 2004, pp. 391-405.
D "Musulmans de Chine et situation internationale (19992003)," La Revue des deux Mondes, January 2004, pp. $42-54$.

D "Chinese Muslim Women: From autonomy to dependence," in S. A. Dudoignon (ed.), Devout Societies vs. Impious States? Transmitting Islamic Learning in Russia, Central Asia and China, through the Twentieth Century, Berlin, Klaus Schwarz Verlag, 2004, pp. 91-104.

D "Islam's Adaptation in China: Present Realities," in The Macau Ricci Institute (ed.), Religion and Culture. Past Approaches, Present Globalisation, Future Challenges (International Symposium - Macau, November 28 $8^{\text {th }}-29^{\text {th }} 2002$ ), Macau, Macau Ricci Institute, 2004, pp. 259-264.

D "Chinese Speaking Muslims (Dungan) in Central Asia: A Case of Multiple Identities in a Changing Context," Asian Ethnicity, vol. 6, no. 2, June 2005, pp. 121-134.

D "Les musulmans de Chine entre dynamisme et contrôle politique," in collaboration with L. Chebbi, C. H. HalfonMichel, Les cahiers de l'Orient, no. 79, 2005, pp. 147-158.

D "Turkestan oriental," Outre-Terre, no. 15, 2006, pp. 409417.

D "Usages de la frontière ; le cas du Xinjiang (XIXe- $x x^{e}$ siècles)," Extrême-Orient, Extrême-Occident, no. 28, 2006, pp. 127-146.

D "19 shijimo he 20 shijichu Faguo wenxianzhong de Zhongguo de musilin yanjiu" 十九世纪末和二十世纪初法国文献 中的中国的穆斯林研究 (Studies on China's Muslims through French texts from late $19^{\text {th }}$ and early $20^{\text {th }}$ century), Shehui kexueyuan, Beijing, 2007.

D "Notes sur la Zhehelinye (Jahriyya) au Xinjiang," Études orientales, no. 25, 2008, pp. 211-222.

D "La mosquée chinoise : support d'identités et de modernités," Revue des mondes musulmans et de la Méditerranée, no. 125, 2009, pp. 109-119.
D "L'islam dans le monde chinois," in M. Gilquin (dir.), Atlas des minorités musulmanes en Asie méridionale et orientale, CNRS éditions, 2010, pp. 267-289.

D "Henan Sanpocun maopi rouzhi lishi chongjian de jige yuansu 河南桑坡村毛皮鞣制历史重建的几个元素 (Elements for a history of fur trade in Henan's Sanpo Village), in C. Lamouroux (ed.), Zhongguo jinxiandai hangye wenhua yanjiu 中国近现代行业文化研究 (Professional cultures in modern and contemporary China), Beijing, Guojia tushuguan chubanshe, 2010, pp. 187-199.

1) "Être Musulmans chinois (Hui) : orthopraxie et orthodoxie: quelles évolutions?", in D. Aigle, I. Charleux, V. Goossaert, R. Hamayon (eds.), Miscellanea Asiatica. Mélanges en l'honneur de Françoise Aubin, Sankt Augustin, Monumenta Serica, 2010, pp. 251-269.

D "Des musulmans de langue chinoise entre Chine et Asie centrale," Revue Relations internationales, no. 145, PUF, 2011, pp. 105-117.

D "Un orientalisme intérieur: les nationalités minoritaires en Chine," in François Pouillon and Jean-Claude Vatin (eds.), Après l'orientalisme. L'Orient créé par l'Orient, Paris, IISMM/Karthala, 2011, pp. 245-252.

D "Pouvoir religieux et pouvoir profane en islam chinois au XXe siècle," in Denise Aigle (dir.), Les autorités religieuses entre charisme et hiérarchie ? Approches comparatives, Turnhout, Brepols, 2011.

D "Choses vues à Yiwu," Outre-Terre. Revue européenne de géopolitique, 2011, no. 30 (Special issue entitled: "Did you say Chinafrica?"), pp. 411-412.

* Selected bibliography from http://cecmc.ehess.fr/document. php?id=116 